Musées, Patrimoine et Culture scientifiques et techniques

$115 \mid 2008$

janvier - février 2008

\title{
Se rendre au musée en famille
}

\section{Anne Jonchéry}

URL : http://journals.openedition.org/ocim/264

DOI : $10.4000 /$ ocim.264

ISSN : 2108-646X

Éditeur

OCIM

Édition imprimée

Date de publication : 1 février 2008

Pagination : 4-14

ISSN : 0994-1908

Référence électronique

Anne Jonchéry, "Se rendre au musée en famille », La Lettre de I'OCIM [En ligne], 115 | 2008, mis en

ligne le 10 novembre 2010, consulté le 19 avril 2019. URL : http://journals.openedition.org/ocim/264 ; DOI : 10.4000/ocim.264 


\section{Se rendre au musée en famille}

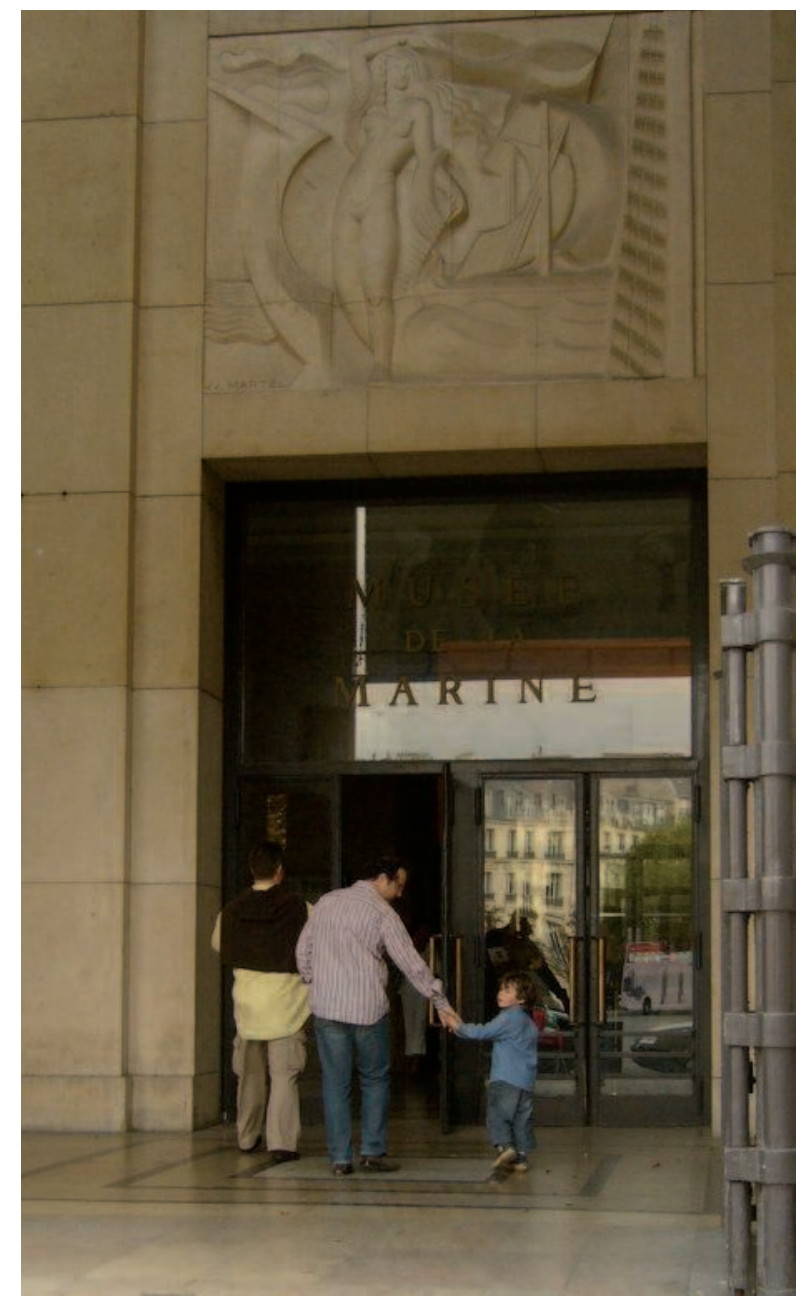

Entrée du musée de la Marine (C) A. Jonchéry

* Anne Jonchéry est docteur en Muséologie des Sciences naturelles et humaines du Muséum national d'Histoire naturelle, assistante de documentation au département des Galeries et enseignante en Muséologie en master 1 au département de Médiation culturelle de l'université Paris 3 - Sorbonne Nouvelle jonchery@mnhn.fr

\section{Anne Jonchéry *}

Venir en famille au musée. Que signifie cet acte et qui concerne-til ? Que dit-il sur la place, le sens de l'institution muséale ? Qu'est-ce qui se joue en matière d'accès au musée et dans les rapports à la culture quand la famille vient au musée? Une analyse du public familial de trois musées et de ses motivations permet à l'auteur de répondre à ces interrogations.

\section{De la famille au musée : un cadre de recherche (1)}

Longtemps considéré comme un acte solitaire, visiter un musée s'inscrit en réalité dans de nombreux contextes de sociabilité : les visiteurs viennent en groupe, entre amis, en couple, en famille, dans les musées de sciences et techniques, d'histoire et de société mais aussi dans les musées d'art. À la lumière des enquêtes de fréquentation, le public familial est en progression constante, notamment dans les musées et centres de culture scientifique. "Élément naturel et fondamental de la société » (2), la famille est à la fois un jalon et un microcosme révélateur du social. Les mutations qu'elle a connues depuis les années 1960 ne remettent pas en cause son statut d'institution sociale primordiale et ses transformations reflètent les évolutions des sociétés modernes. Pour ces raisons, nous avons choisi de questionner l'acte de visiter une exposition en famille, révélateur en puissance des relations des individus au musée, des usages de celui-ci et de son positionnement dans le monde social. 
Au sein de la recherche en Muséologie, l'étude du public familial a connu une expansion tant géographique qu'historique. La visite en famille fait l'objet de recherches en Amérique du Nord dès la fin des années 1970, avant que des chercheurs ne s'y intéressent en Europe, en Angleterre d'abord puis en France depuis le début des années 1990. Initiées par les universités ou par les institutions muséales, ces recherches se déroulent de manière privilégiée dans des musées et centres scientifiques. Elles portent principalement sur l'apprentissage et le comportement des familles durant la visite, moins sur le sens d'une telle démarche. Ces études révèlent par ailleurs l'hétérogénéité et l'approximation des définitions du public familial : le terme même recouvre des réalités variées. Face à ce constat, nous avons cherché à établir une photographie du public familial et à saisir ce qui se joue quand la famille vient au musée.

\section{L'Amour de l'art face aux évolutions familiales et muséales actuelles}

À la fin des années 1960, les travaux de Bourdieu et Darbel ont montré que la visite de musée était une pratique socialement construite, déterminée par le niveau d'études et par les caractéristiques sociales des individus. Dans L'Amour de l'art (1966), la pratique de visites apparaît comme une pratique de distinction, l'apanage des classes cultivées et dominantes, s'apparentant à un habitus culturel, principe structuré par la position sociale et structurant son style de vie. Dans ce cadre, la famille est considérée comme l'instance de socialisation à cet habitus : elle est l'organe de transmission de la pratique de visites. L'approche de la famille en Muséologie a été modelée par ce cadre de transmission issu des travaux de Bourdieu. Nous avons voulu ici tester la pérennité de cette analyse car la famille et le musée ont connu de profondes mutations.

La famille contemporaine s'est transformée et ne ressemble pas à la famille des années 1960. Elle s'est modifiée dans ses formes mais elle a surtout évolué dans sa nature et ses fonctions (Singly, 1999). La famille contemporaine est relationnelle et individualiste, manifestant un repli autour de la famille conjugale - groupe domestique formé par le ou les conjoints et/ou le ou les enfants. Son fonctionnement interne nécessite relations et communication afin de réguler les intérêts des individus et ne léser personne. Ses fonctions évoluent : autrefois chargée directement de la reproduction sociale, elle se trouve dépossédée de ce pouvoir par l'école qui, par le diplôme, donne la valeur sociale de l'individu. La famille agit néanmoins indirectement sur cette reproduction en développant des stratégies éducatives pour favoriser la réussite scolaire. Mais elle doit aussi concilier cette fonction avec une fonction plus récente, celle de la construction identitaire et de l'épanouissement de l'enfant, liée à la diffusion du discours psychologique et à une société d'économie de marché de plus en plus individualiste.

L'offre muséale s'est aussi transformée. La " fièvre muséale » (Benhamou, 2000) a touché Paris et les régions, voyant fleurir à partir des années 1980 de nombreux musées aux spécialités plus variées que précédemment, et plus soucieux du public. Les musées de société, les écomusées, ou même les différentes spécialités apparues au sein des musées d'art témoignent de cette diversification. Les grands programmes de rénovations et de créations de musées comme la politique des Grands Travaux ont accéléré ce mouvement. Le paysage muséal actuel se présente pluriel, mouvant et multiple (Eidelman et Van-Praët, 2000), difficilement comparable à celui plus monolithique, des années 1960.

L'appréhension de ces évolutions amène à s'interroger sur la pérennité de l'analyse de Bourdieu et sur les nuances à y apporter. À travers nos hypothèses il s'agit de complexifier le regard sur une visite en famille uniquement envisagée comme une pratique de transmission, réservée à une élite, et d'imaginer des visites familiales répondant à d'autres injonctions et à d'autres enjeux, liés au fonctionnement de la famille contemporaine. En quoi le contexte familial ne permettrait-il pas, par ses caractéristiques relationnelles, un accès assoupli des individus aux établissements muséaux ? Un accès moins dépendant des pratiques antérieures des adultes par exemple et plus autonome de leur niveau de diplômes, voire plus " démocratisé »? Nous émettons l'hypothèse que, dans une institution en mutation, il pourrait se jouer autre chose lors de la visite en famille qu'une simple transmission de pratiques, de l'ordre du partage, de la découverte collective.

Les travaux de Lahire (2004) sur la dissonance des profils culturels et sur les marges de « liberté » des choix culturels de l'individu, les études d'Eidelman (2005) sur l'imprévisibilité des « carrières » de visiteurs renforcent nos hypothèses quant à une pratique de visite socialement moins déterminée. L'analyse de Certeau (1980) a montré tout l'intérêt d'étudier les usages, les contextes d'utilisation des équipements culturels, en partant plutôt de ce que les gens en font que de l'utilisation prévue par les fabricants. Ainsi, pour éprouver nos hypothèses, 
nous avons étudié les profils des groupes familiaux visiteurs, leurs contextes de visites, leurs démarches et motivations, dans trois établissements différents.

Afin d'appréhender des réalités différentes de la visite en famille, nous avons diversifié les terrains d'enquêtes et croisé les méthodes d'enquêtes. Trois musées parisiens aux spécialités différentes - sciences, art et histoire - ont été choisis : les Galeries de Paléontologie et d'Anatomie comparée du Muséum national d'Histoire naturelle, le musée d'Orsay et le musée national de la Marine. Une enquête quantitative par questionnaire s'est déroulée auprès de 350 groupes familiaux rencontrés à l'entrée des trois musées, visant à recueillir pour chaque établissement le profil de la fréquentation familiale, la composition des groupes et leurs caractéristiques sociales. Une enquête qualitative par entretien semidirectif a permis la constitution d'un corpus de 112 entretiens de groupes familiaux venus visiter l'un des trois musées, abordant les contextes, démarches et motivations de leur visite.

\section{Richesses et complexités de la visite en famille : des résultats nuancés}

\section{Du mirage d'une fréquentation familiale} démocratisée à des processus individualisés L'hypothèse d'une fréquentation familiale moins marquée par les inégalités sociales que la fréquentation générale, se fondait sur le profil des visiteurs globalement moins élitiste dans les musées recevant un public familial important (Mironer, 2001). Notre comparaison au sein de chacun des trois musées, entre la stratification sociale des groupes familiaux interrogés et les données correspondant à l'ensemble de leur public, ne corrobore pas cette hypothèse de démocratisation. En effet, la visite en famille est empreinte des mêmes inégalités sociales que la fréquentation générale : on retiendra le poids de la certification (56\% des adultes ont un niveau supérieur ou égal au bac), la majorité d'individus CPIS (3) (36\%) et PI (4) (29\%) et la quasi-exclusion des ouvriers $(2 \%)$ de la fréquentation familiale, traits identifiés par Bourdieu à l'échelle de l'ensemble des publics de musées.

L'hypothèse d'une mixité sociale au sein des groupes ne se réalise que très peu, compte tenu de limportance des groupes comprenant un seul adulte (53\%), de la domination des groupes parent(s)/enfant(s) (75\%), et de la forte homogamie au sein des couples parentaux. On remarque néanmoins une diminution des niveaux bac +5 au profit des bac $+1+2$ et un tassement des CPIS au profit des PI quand les parents

\section{Le Jardin des Explorateurs
Un nouveau projet pour les familles}

Le Jardin botanique et l'Insectarium de Montréal font partie, avec le Biodôme et le Planétarium de Montréal, des Muséums nature de Montréal. Le Jardin botanique de Montréal a été réalisé en 1931 et accueille annuellement 900000 visiteurs, dont 65000 enfants en visite scolaire. L'Insectarium de Montréal est établi sur le site du Jardin botanique depuis 1990 et accueille à lui seul 350000 visiteurs par année. Les Muséums nature ont pour mission de faire connaître la nature et les savoirs qui s'y rattachent, de contribuer à l'étude et à la préservation de la biodiversité et de promouvoir des comportements responsables face à l'environnement.

Depuis 2001, les Muséums nature de Montréal ont amorcé une planification afin d'identifier leurs stratégies de développement et leurs priorités pour les années 2002 à 2006. Dans le cadre de cette planification, une réflexion, tenue à l'automne 2005, a permis de dégager le potentiel de développement pour la clientèle familiale et les enfants sur le site du Jardin botanique et de l'Insectarium de Montréal. Lidentification de pôles d'attraction pour la famille et les enfants s'est notamment appuyée sur une vaste enquête menée auprès de la clientèle des Muséums nature : Étude sur les besoins, les attentes et la satisfaction de la clientèle - Profil global des Muséums nature de Montréal. Cette étude révèle, si l'on combine la basse et la haute saison, que $37 \%$ des visiteurs du Jardin botanique sont accompagnés d'enfants, ce pourcentage s'élevant à $49 \%$ pour l'Insectarium de Montréal.

Confirmant ainsi sa clientèle majoritairement familiale et étant identifié par d'autres sondages comme l'un des musées favoris des enfants, l'Insectarium de Montréal a élaboré en 2006 un Plan directeur Diffusion et mise en valeur 2006-2011 qui vient synthétiser l'ensemble des priorités et des actions pour les cinq prochaines années. Le pourtour de l'Insectarium représente une zone sous-exploitée qui mérite une intervention à court et moyen terme. Elle comporte actuellement une aire de jeux pour enfants qui est constamment utilisée bien que fort désuète. Cette zone compte également plusieurs avantages qui justifient d'y développer une offre enfants/familles plus complète et plus attrayante : un stationnement à proximité, un positionnement stratégique par rapport aux autres pôles d'attraction du Jardin botanique (les jardins culturels) et une aire de restauration. Il est également important de souligner que la clientèle majoritaire du Jardin botanique y vient (en haute saison, à $60 \%$ ) pour se relaxer, passer une belle journée à l'extérieur et se divertir. Le développement de ce pôle d'activités au pourtour de l'Insectarium se fait donc en complémentarité et dans le respect des attentes de cette clientèle acquise et fidèle, qui ne recherche pas la même expérience de visite. 


\section{au Jardin botanique et à l'Insectarium de Moniréal}

C'est donc à partir de ces constatations que les Muséums nature de Montréal ont entrepris de développer le projet du Jardin des Explorateurs, afin de créer un pôle d'attraction majeur et d'améliorer l'offre éducative et les services pour la clientèle familiale et les enfants. Ce projet développé par la firme d'architecture de paysage et de muséographie Schème au cours de l'année 2006-2007, est constitué de quatre aires extérieures adjacentes qui formeront un tout nouveau secteur du Jardin botanique de Montréal.

\section{Le Labyrinthe des découvertes}

Un tout nouveau labyrinthe en forme spectaculaire de feuille lancéolée sera dédié au thème de la biodiversité végétale. Les installations éducatives qu'on y trouvera visent à intéresser plus particulièrement les enfants de 8 à 12 ans. La forme des chambres du labyrinthe est inspirée des cellules qui composent une feuille et celle des sentiers rappelle ses nervures. Le labyrinthe est doté d'un belvédère d'observation, il est divisé en quatre parties thématiques liées aux plantes : « Nourrir » (importance des plantes en tant que principale source alimentaire), « Abriter » (utilité des plantes pour abriter humains et animaux), « Évolution des plantes terrestres », "Protéger la biodiversité ». Chacune des quatre chambres principales proposera des activités et des jeux de découverte du monde végétal et de la biodiversité.

\section{Sur la piste des insectes}

Ce tout nouveau Jardin des insectes du Québec et son kiosque d'animation extérieure (L'Agora), comportera une volière à papillons, des activités éducatives, un parcours de découvertes à travers trois habitats différents : un pré fleuri, un étang et un bois. Il sera divisé en six sections, chacune associée à un ordre d'insectes du Québec. Deux parcours d'exploration y seront proposés, chacun bordé de différents éléments d'interprétation : des insectes naturalisés présentés sous globe d'acrylique clair ; une petite volière à papillons, incluant un jeu d'association des stades de métamorphose des papillons ; un étang équipé d'une lunette d'observation d'insectes aquatiques ; une agora pour les animations ; cinq jeux interactifs d'imitation d'insectes (grillon, sauterelle, abeille, libellule, coléoptère).

\section{Terre de jeux}

Cette aire de jeux inédite sur le thème des nids d'insectes sera composée de deux sections qui répondent aux besoins de deux groupes d'âge d'enfants : les 18 mois à 5 ans et les 6 à 12 ans. Elle comprendra cinq types de jeux : « Dômes d'insectes », «Alvéoles d'une ruche en 3D », « Coupoles de larves d'abeilles », « Nid bulle d'air », « Toiles d'araignées ».

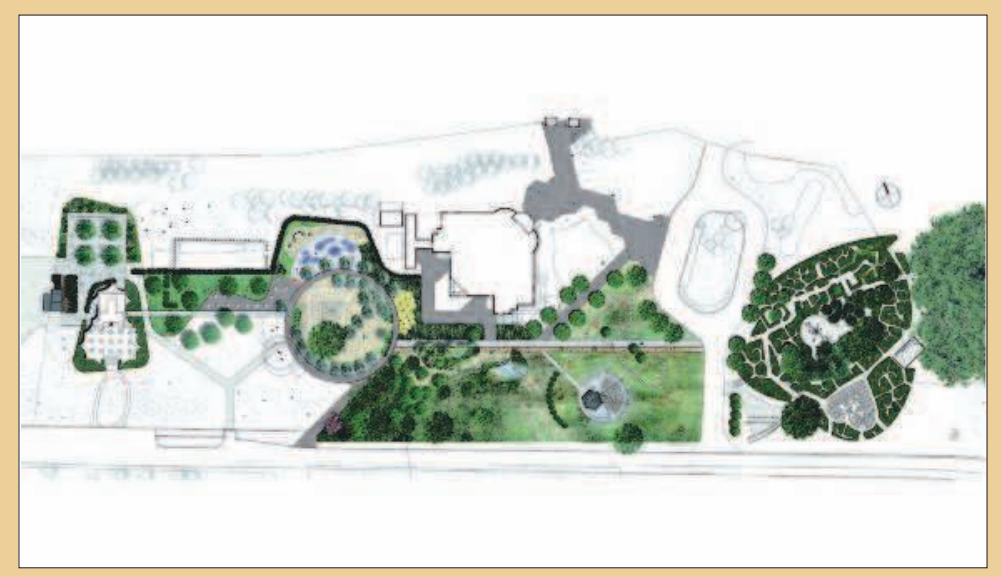

Positionnement des aires du projet sur le site du Jardin botanique avec de gauche à droite : I'aire de pique-nique, l'aire de jeux, le Jardin des insectes du Québec, le Labyrinthe végétal. (c) Muséums nature de Montréal

\section{Le Resto-terrasse L'Amiral}

On créera une nouvelle aire de pique-nique pour les familles et deux terrasses seront réaménagées autour du restaurant du Pavillon Fuji, près de l'Insectarium. Initialement, ce restaurant extérieur pouvait accueillir 80 convives. Une fois réaménagé, le nombre de places assises passera à 240. De plus, afin de mieux lier cette aire à l'ensemble des aires du Jardin des Explorateurs, des possibilités d'apprentissage ludique sur le thème des fleurs insectivores et des pièces buccales d'insectes seront offertes aux visiteurs sous la forme d'intrigants panneaux à bascule proposant chacun une devinette.

Conjointement coordonné par la direction de l'Insectarium de Montréal et celle du Jardin botanique de Montréal, porté par l'ensemble du personnel des deux institutions et notamment Francine Hoffman (Jardin botanique), Elaine Boileau, (Insectarium) et Louise Lalonde (Jardin botanique et Insectarium), le projet du Jardin des explorateurs est en cours de développement. Les Muséums nature de Montréal collaborent depuis décembre 2006 avec la firme Schème pour élaborer le concept et le design des quatre aires pour enfants et familles. Cette étape est terminée et nous nous apprêtons à lancer les appels d'offres pour la réalisation finale du projet qui devrait intervenir au printemps 2009.

JOHANNE LANDRY Directrice de l'Insectarium de Montréal, Muséums nature de Montréal 
viennent en couple, si on les compare aux parents venus seuls avec leur(s) enfant(s). Pour les professions et certifications intermédiaires, être en couple aurait une dimension de réassurance facilitant la visite en famille de musées.

Les trois musées témoignent chacun de certaines spécificités : le musée de sciences apparaît un peu moins élitiste, avec une hétérogénéité de catégories socioprofessionnelles et de diplômes plus importante au sein des groupes. Le musée d'art en revanche concentre une fréquentation familiale très élitiste et peu mélangée. Tandis que le musée d'histoire polarise une majorité de groupes très diplômés et CPIS, et une part un peu plus importante de groupes plus populaires et moins certifiés.

Si l'hypothèse d'un public familial plus démocratisé s'avère peu concluante, la visite en famille ne s'inscrit pas pour autant dans un processus de transmission de pratiques : pour une part des groupes, la visite familiale ne se conjugue pas à une familiarité muséale des parents (14\% s'y rendaient moins d'une fois par an, $28 \%$ une à deux fois par an). De plus, la moitié des parents déclarent ne pas avoir bénéficié de pratiques enfantines. Mais surtout, à l'échelle des entretiens, le contexte familial génère des pratiques de visites chez des adultes non visiteurs de musées, pratiques rendues possibles par la parentalité. Il semble notamment que les motivations de la venue au musée - visant les bénéfices personnels et éducatifs de l'enfant - attachent la visite au présent et au futur. L'investissement de ces deux temporalités faciliterait l'accès aux musées des adultes, contribuant à les affranchir d'une certaine timidité sociale, à surmonter les obstacles culturels. La parentalité ouvre ainsi de nouvelles potentialités dans les rapports aux musées et dans les horizons de visites, réactualise et réaménage les pratiques muséales.

Un reflet des évolutions

de la famille contemporaine

La photographie du public familial des trois musées révèle la prépondérance du lien de filiation avec $75 \%$ de groupes composés uniquement de parent(s) et de leur(s) enfant(s) : ce résultat reflète le repli sur la famille conjugale montré par la Sociologie (Singly, 1999). Pour autant, $57 \%$ des groupes parent(s)/ enfant(s) ne comprennent qu'un seul parent témoignant du fonctionnement relationnel et assoupli de la famille contemporaine qui réalise ses loisirs sous des formes de plus en plus fractionnées (un parent et un enfant).

Dans l'enquête qualitative, la visite apparaît comme un accomplissement des fonctions de la famille

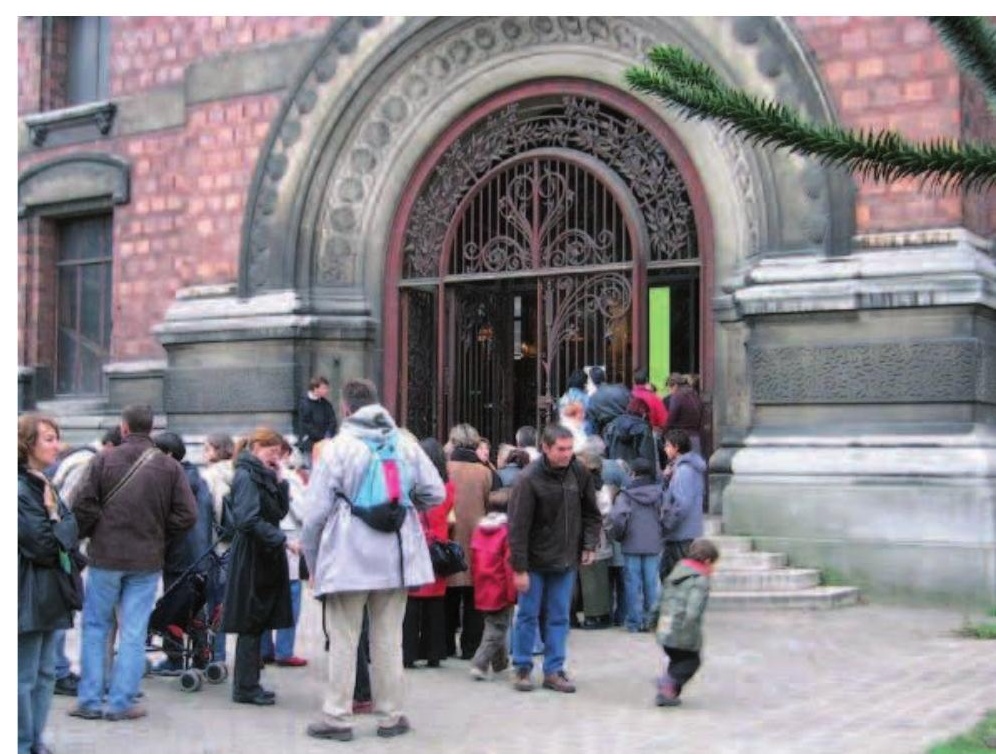

File d'attente au Muséum national d'Histoire naturelle (c) A. Jonchéry

contemporaine. La motivation d'apprentissage conjuguée aux interventions plurielles de l'école reflète une mobilisation scolaire importante, chez une grande partie des groupes familiaux. Ce résultat confirme l'hypothèse initiale : la visite en famille s'affirme comme un investissement éducatif des parents, visant notamment la réussite scolaire. Ce phénomène répond à l'évolution des modes de reproduction sociale. Néanmoins, la visite de musées revêt encore le statut d'habitus culturel : pour une part des familles, elle s'inscrit dans un mouvement de transmission d'un capital culturel, corroborant la théorie de Bourdieu. Ce processus perdure donc mais n'est ni unique, ni majoritaire. Des visites familiales répondent aussi au plaisir de l'enfant et à son développement personnel, participant de l'autre fonction de la famille contemporaine visant la construction identitaire de l'enfant.

Les formes et logiques de visites familiales reflètent les évolutions de la famille contemporaine. Les musées apparaissent ainsi comme des miroirs des évolutions sociales : la famille s'approprie les institutions muséales et les adapte à ses nouveaux fonctionnements. Mais cette perméabilité au social est rendue possible grâce à la pluralité du champ muséal : les musées de sciences et d'histoire dévoilent plus directement ces changements sociaux tandis que les musées d'art font preuve d'une certaine résistance - avec plus de groupes comprenant toute la famille nucléaire, et plus de visites liées à la transmission d'un habitus. 
D'une prolifération de contextes de visites à la construction d'une pratique familiale

La visite familiale s'inscrit dans des contextes variés : les provenances géographiques et les activités diverses associées à la visite créent une variété de contextes de fréquentation étonnante. Le jeu dynamique des musées (chaque musée ayant ses spécificités) se croise avec celui du public et de chaque visiteur. Cette diversité de contextes témoigne du caractère malléable et flexible de l'institution muséale dont la visite s'ajuste, de manière singulière et personnelle, à l'emploi du temps des familles.

Mais au-delà de cette inventivité à l'échelle de la visite, un processus fédérateur se dégage à l'échelle de la pratique de visites : celui d'une pratique en construction dans le temps. La pratique familiale de visites de musées est à la fois linéaire, balisée dans le temps par l'âge des enfants, et discontinue, interrompue puis reprise dans le cas des familles nombreuses ou recomposées. Marquée par des phénomènes de ruptures, liés notamment à la naissance des enfants, elle évolue en fonction du cycle de développement de la famille. La pratique revêt aussi une dimension cyclique, suivant des temps sociaux comme celui des vacances scolaires, ou suivant des fréquences plus régulières, mensuelles par exemple.

Ce caractère cyclique n'induit pas pour autant reproduction à l'identique : quand la visite se renouvelle, la famille a l'expérience de ses visites antérieures. En évolution, la pratique familiale s'apprend et se construit par étapes. Les premières visites se déroulent souvent dans les musées de sciences, considérés comme les plus adéquats pour familiariser les enfants à ce type de lieu. Quand les enfants grandissent, les musées d'art peuvent alors être investis. La pratique familiale et le mode de visite se modifient, suivant les évolutions et transformations du groupe familial, des individus et de leur familiarité.

Au fil des entretiens, la visite familiale apparaît aussi dans ses difficultés et ses embûches. Deux difficultés coexistent : l'une inhérente à l'équipement muséal (espace contraignant et codifié) l'autre propre à l'acte familial (acte collectif rarement le fruit d'un consensus immédiat). Des stratégies de compensation et des négociations sont mises en œuvre pour surmonter ces obstacles. Il demeure que, derrière l'image du bonheur familial au musée, se dessine une réalité plus complexe.

Logiques et motivations de la visite en famille À la lumière des entretiens, la visite familiale combine plusieurs motivations : il s'agit d'une part d'un acte éducatif, lui-même polysémique. Le musée est considéré comme un lieu de connaissance, d'éveil et d'ouverture au monde ; il est appréhendé dans ses spécificités, pour le rapport physique à l'objet et à la culture matérielle qu'il permet. Les relations entre l'école et la visite peuvent être de l'ordre de la prescription inconsciente ou viser la réussite scolaire : les implications de l'institution scolaire à de multiples degrés de la visite reflètent à la fois le poids de l'école dans la famille et ses connexions avec l'institution muséale.

À ces motivations éducatives s'articule l'intention de faire plaisir à l'enfant et de contribuer à son épanouissement personnel, de l'aider à trouver ses intérêts. Cette motivation répond à l'injonction d'une société individualiste dans laquelle l'individu doit s'autonomiser, devenir lui-même. Néanmoins les parents souhaitent certes favoriser l'épanouissement de l'enfant mais par une activité culturelle qui contribue à son développement intellectuel. Cette ambiguîté corrobore l'analyse plus globale des pratiques culturelles familiales de Singly (2003), soulignant la position instable des parents, lesquels oscillent entre attention, respect des envies de l'enfant, et exigence pédagogique.

La convivialité participe d'autre part très fortement des motivations de la visite familiale, confirmant les enquêtes européennes et nord-américaines sur l'importance de la sociabilité au musée. L'organisation de la vie quotidienne, le rythme et la conciliation des temps sociaux et professionnels expliquent notamment cette attente de convivialité : la rareté des moments passés en famille génère des exigences de qualité. Parfois motif exclusif de la visite, la convivialité est associée dans la majorité des familles à la notion d'expérience partagée et de dialogue dont l'exposition est le support. Celle-ci transparaît aussi comme un dispositif d'exploration et de connaissance de l'autre. Propulsé dans un environnement inédit, l'enfant se comporte différemment que dans l'univers domestique. La visite familiale offre ainsi l'occasion au parent de découvrir son enfant, ses centres d'intérêts, des facettes de son identité. Les bénéfices de cette observation s'inscrivent alors dans la relation familiale, dépassant le temps et l'espace de la visite.

L'intérêt personnel de l'adulte participe de manière secondaire ou accessoire à la visite : il apparaît parfois complexe à articuler à la visite en famille, sujet de frustrations quand il ne peut être satisfait. Il est révélateur du positionnement identitaire de l'adulte qui se présente d'abord comme « parent ».

La visite familiale se révèle in fine un acte producteur de divers registres identitaires : elle demande un positionnement culturel de chaque individu, 
En famille au musée :

Essai de mise en pratique dans la définition d'un espace d'exposition permanente

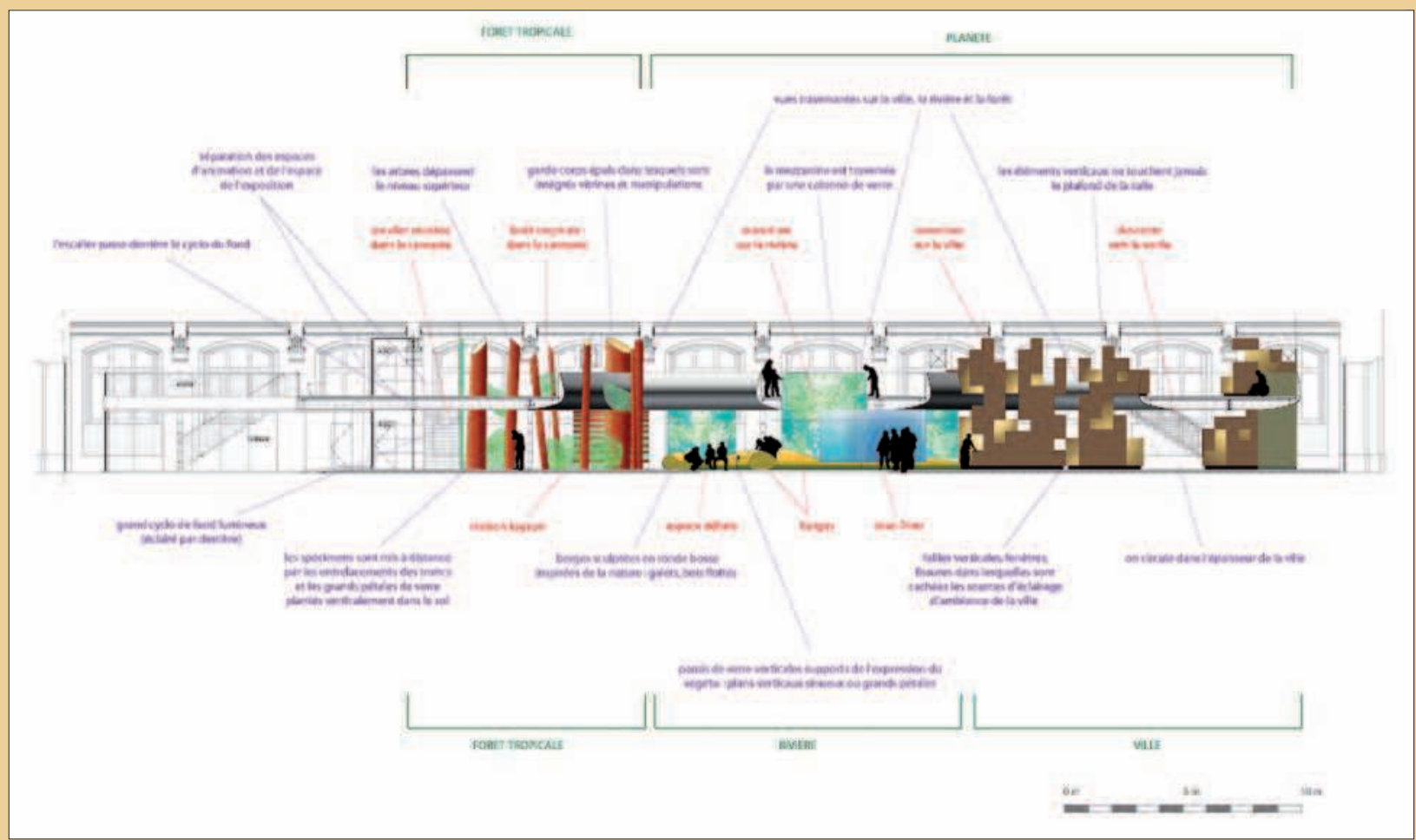

Coupe longitudinale de l'espace montrant dans la partie droite l'espace d'exposition et dans la partie gauche les deux salles d'activités. L'espace d'exposition est organisé en deux parties principales :

au rez-de-chaussée, "Biodiversité », "Menaces » et "Actions » dans trois milieux, successivement la ville, la rivière et la forêt tropicale; à l'étage, les mêmes thèmes sont abordés à l'échelle de la planète.

๑) P. Payeur/E. Lefrançois/M. Hardel/C. Le Bihan/A. Robaglia

Depuis 1994, année d'ouverture de la Grande Galerie de l'Évolution (GGE), les études réalisées sur le site par l'Observatoire permanent des Publics ${ }^{(1)}$ révèlent une augmentation régulière de la proportion du public familial : de moins de $30 \%$ du public individuel en 1995, celui-ci avoisine les $50 \%$ depuis 2000. Cette tendance suit un mouvement actuel général dans les musées et justifie que ceux-ci portent une attention particulière au public familial.

Parallèlement s'est révélé un certain manque dans l'offre de la GGE vis-à-vis des enfants d'âge moyen. Le spectacle des animaux naturalisés émerveille toujours les plus petits, alors que la richesse des contenus portés par les balcons périphériques répond à la curiosité des plus grands. Par contre, vis-à-vis des enfants d'âge correspondant grosso modo à l'école primaire, les informations sont rares dans la première partie de l'exposition puis complexes et difficiles d'accès dans les parties suivantes, et ce d'autant plus que ces enfants sont demandeurs d'autonomie de visite. L'espace conçu à leur intention, la « salle de découverte ", occupe une surface réduite et est situé en dehors du parcours principal de visite. De fait, les études de public révèlent qu'il passe inaperçu pour beaucoup de visiteurs et ceux qui visitent cet espace en ressortent peu satisfaits.

Laugmentation de cette catégorie de public et la nécessité pour l'institution de s'y adapter a conduit le Muséum national d'Histoire naturelle à mettre en œuvre un nouvel espace d'exposition permanente pour les enfants au sein de la Grande Galerie de l'Évolution (2). Une question essentielle s'est alors rapidement posée : exposition pour l'enfant seul ou pour l'enfant accompagné - et en particulier de ses parents ? Deux opinions diamétralement opposées peuvent être mises en avant. L'une plaide pour l'autonomie, souhaitant d'abord répondre à la demande exprimée par l'enfant et soutenir sa démarche personnelle ${ }^{(3)}$. L'autre met en avant d'une part les motivations à la visite exprimées par la cellule familiale dans son ensemble (4), d'autre part l'intérêt de la « coéducation » ou encore de « l'éducation informelle » dans le contexte particulier du musée et de l'exposition ${ }^{(5)}$. 
En effet, l'une des principales motivation à la venue au musée en famille est le fait de se retrouver ensemble, de partager une activité commune, de se découvrir en dehors du cadre domestique. Par ailleurs, la coéducation au musée est reconnue comme contribuant à l'enrichissement de l'événement de visite comme à la socialisation de l'enfant ou à un partage égalitaire entre enfant et adulte de la connaissance et du plaisir d'apprendre.

C'est ce contexte de visite en famille qui a été privilégié dans l'élaboration du nouveau projet. De la même façon a été proposée la présence permanente d'une petite équipe d'animation chargée d'accompagner les groupes scolaires mais aussi, sous forme facultative, les visiteurs individuels. Cette équipe serait également chargée de définir et de mettre en œuvre de courtes animations, dites " animations-flash », sur le lieu même de l'exposition, s'inspirant des pratiques de divers musées comme la Cité des Enfants ou l'Explor@dome à Paris (6).

Les principes ayant conduit à la définition du projet et de ses formes de médiation se sont élaborés progressivement à partir de résultats de travaux de recherche et de réflexions théoriques mais aussi de choix pragmatiques inspirés d'expériences et de réalisations existantes ou issus de la sensibilité des différents membres de l'équipe projet, de lidentité de l'institution, ou encore des demandes de sa direction. Ils sont actuellement en cours de matérialisation sous forme d'un programme muséologique et d'un projet scénographique détaillés. L'ouverture de ce nouvel espace est prévue pour le printemps 2009.

Didier Julien-LAFERrière Concepteur d'exposition au Muséum national d'Histoire naturelle

\section{Notes}

(1) Études réalisées annuellement de 1995 à 2001 par C. Fromont-Colin puis F. Lafon.

(2) Proposition faite en 2003 par A. Parent qui assure depuis la conduite du projet.

(3) Voir en particulier Singly, F. L'art et la manière de considérer un enfant dans un musée, colloque pARTages : quelles expositions d'art pour les enfants ?, musée du Louvre, 28 avril 2006. Actes téléchargeables sur www.louvre.fr

(4) Jonchéry, A. Quand la famille vient au musée : des pratiques de visites aux logiques culturelles. Thèse de doctorat en Muséologie, Muséum national d'Histoire naturelle, 2005

(5) Voir par exemple Guichard, J. Nécessité d'une recherche éducative dans les expositions à caractère scientifique et technique, Publics \& Musées, n7, 1995, pp. 95-115 ; Cordier, J.-P. et Serre, S., Interactions familiales au musée : approches sociologiques et psychocognitives, in $L a$ muséologie des sciences et ses publics, de J. Eidelman et M. Van-Praët (dir.), Paris : PUF, 2000, pp. 259-279.

(6) Selon des visites et interviews des membres du personnel de nombreux musées réalisées en 2003 et 2004 pour définir le projet. pour lui-même et par rapport aux autres membres présents ; elle exige de chacun une gestion (régulation, conciliation ou sélection) de ses dimensions personnelles et statutaires. En effet, si la pratique culturelle de visites revêt une fonction de distinction de soi comme l'a montré Lahire (2004), dans la pratique familiale cette fonction se trouve enrichie et complexifiée par la présence de l'autre et par l'identité du groupe. La visite a un sens pour soi, pour l'identité intime de l'individu, sens d'autant plus important qu'il est réfléchi par la présence des autres membres du groupe. Elle a aussi un sens pour son identité statutaire, parentale ou filiale. Enfin, elle est porteuse de sens pour le groupe et la mémoire familiale. Le jeu entre ces différents registres identitaires engendre alors des phénomènes d'accessibilité au musée d'individus initialement non visiteurs, mais aussi des phénomènes de tension, de frustration et de conciliation dans la visite.

\section{De quelques réflexions sur la visite en famille...}

\section{... Autour de la question}

de la démocratisation culturelle

L'étude du public familial des musées s'ancre dans la problématique de la démocratisation de l'accès au musée. Celle-ci a fait l'objet de nombreuses analyses (Passeron, 2003 ; Donnat, 1998) et de discours contradictoires avec d'une part des études qui montrent, enquêtes à l'appui, combien les pratiques culturelles des individus ne sont pas déterminées par leur origine sociale et leur niveau de diplômes mais se révèlent au contraire imprévisibles et dissonantes (Eidelman, 2005 ; Lahire, 2004); et d'autre part les enquêtes Pratiques Culturelles des Français réalisées tous les huit ans par le ministère de la Culture qui dressent toujours le même constat des inégalités sociales d'accès à la culture.

Cette recherche fait surgir une réalité nuancée. À l'échelle quantitative, la composition sociale du public familial montre des phénomènes de reproduction de classe : les inégalités sociales pèsent toujours autant sur les visiteurs de musées, les visiteurs en famille notamment. Les constantes identifiées par Bourdieu (1966) liant la visite de musée à un haut niveau de diplôme et à une PCS élevée apparaissent toujours d'actualité. Néanmoins, les parents concernés n'ont pour moitié pas bénéficié de visites enfantines, écartant les mécanismes de stricte reproduction. De plus, l'enquête qualitative fait apparaître des processus hors des schémas de transmission bourdieusiens. Ces pratiques individualisées révèlent 
les transformations engendrées par la parentalité dans les rapports des individus aux musées. Ainsi, des adultes initialement non-visiteurs et peu diplômés, ont accès au musée par le contexte familial qui légitime leur démarche. La notion même de transmission se trouve à repenser, quand ces parents initient finalement leurs enfants à leur liberté individuelle d'accéder à un équipement culturel (auquel ils n'ont pas été familiarisés). Il se dégage également des entretiens que les musées de sciences et d'histoire facilitent l'entrée au musée de groupes peu familiers de la sphère muséale.

Méthodologiquement, la conjugaison d'enquêtes quantitative et qualitative a permis d'articuler deux regards et d'identifier d'une part des phénomènes de

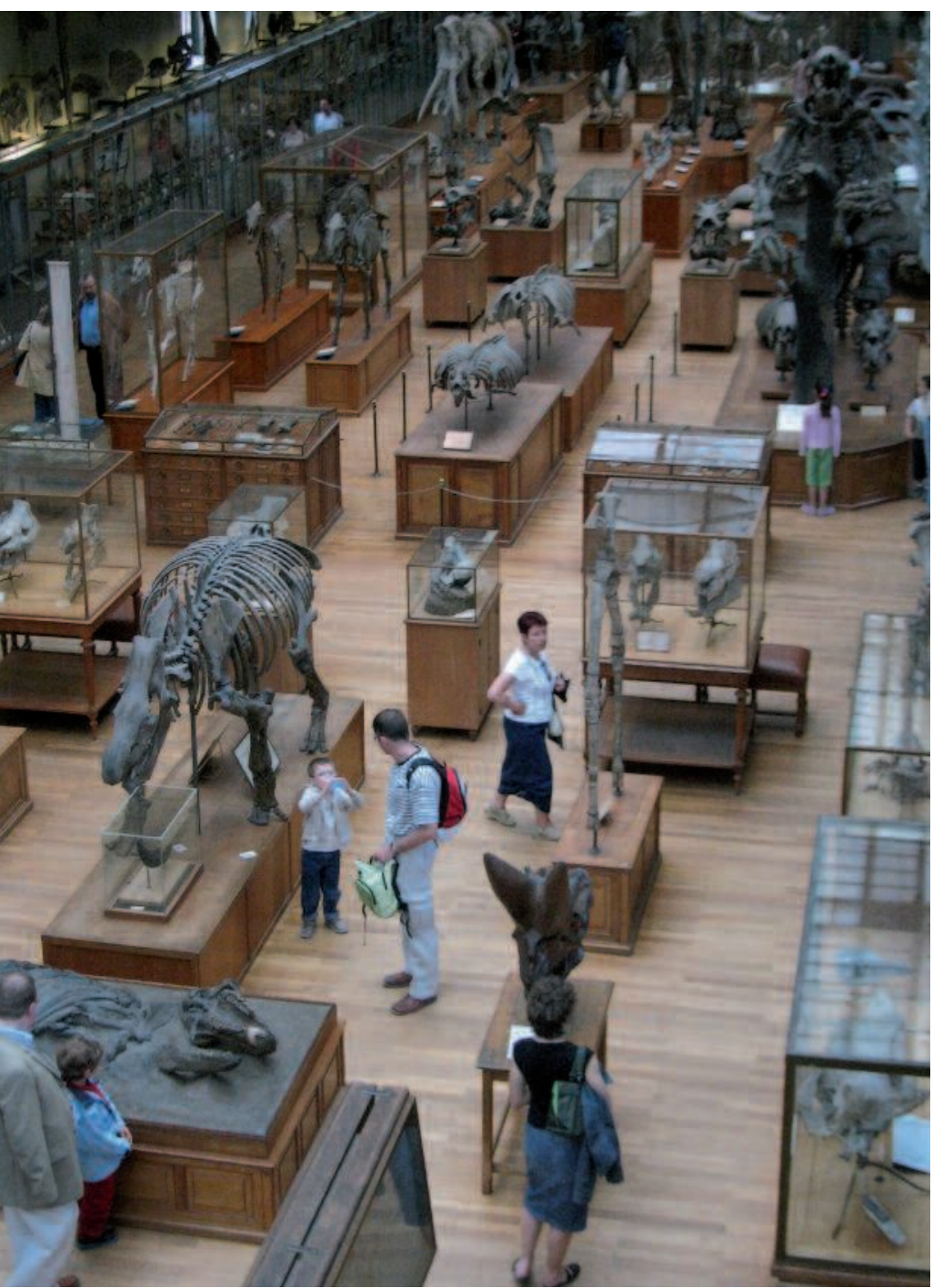

Parents et enfants à la Galerie de Paléontologie et d'Anatomie comparée du Muséum national d'Histoire naturelle () A. Jonchéry société globaux, de reproduction de classe, et d'autre part des pratiques individualisées au sein des classes et des familles, qui questionnent et complexifient les premiers phénomènes.

L'enquête quantitative donne une photographie de la fréquentation familiale et de ses caractéristiques, photographie d'une réalité sociale de l'institution muséale, inégalitaire et élitiste, plutôt statique. L'enquête qualitative éclaire quant à elle la réalité des rapports individuels et fait émerger la richesse et la mobilité des pratiques. Les logiques de visites, le réaménagement des rapports au musée des individus devenus parents révèlent un monde en mouvement. La prise en compte préalable des métamorphoses du paysage muséal - par la diversification des terrains d'enquête - renforce ce point de vue. Cette réalité est celle d'un monde mobile, avec les évolutions de la famille et suivant les transformations du musée. Ainsi, l'étude de la démocratisation de l'accès à la culture s'enrichit d'une approche plurielle et l'articulation de plusieurs échelles d'analyse des pratiques culturelles permet de saisir différents aspects d'une réalité complexe.

\section{... Autour de la place et du positionnement des musées dans la société}

Dans une société très médiatisée, où limage et la vidéo deviennent des moyens de communication et d'information dominants, qu'en est-il d'une institution qui conserve des objets patrimoniaux, en expose une sélection et les propose comme mode de connaissance ? Mis en balance par de nombreux groupes familiaux avec d'autres médias de communication comme le livre ou la télévision, le dispositif de l'exposition est considéré pour le rapport au savoir très spécifique qu'il permet : la confrontation à l'objet concret, réel, et authentique constitue une expérience unique et propre à la sphère muséale. Cette expérience est recherchée dans la démarche-même de la visite familiale : en interrogeant les groupes sur les activités de substitution à leur visite, plus de la moitié évoque une visite d'un autre musée, attestant de la position privilégiée du musée peu concurrencé par d'autres types d'activités et de loisirs. Ainsi, à l'heure où l'on s'interroge sur la place d'une institution qui place l'objet au centre, les pratiques familiales rassurent quant à sa pérennité.

Par ailleurs, les familles nouent des relations qui leur sont propres avec l'institution muséale. La diversité des contextes observés, des emplois du temps dans lesquels s'insère la visite, témoigne de l'inventivité des pratiques dont la dimension familiale facilite la narration. La pluralité de l'offre muséale semble 
renforcer et élargir le champ des possibles. Ces résultats révèlent le musée dans toute sa flexibilité : loin des représentations figées et sacralisées, les familles entretiennent des rapports souples et dynamiques avec la sphère muséale. Les usages personnalisés des visiteurs attestent ainsi de la flexibilité des musées.

Enfin, notre étude renseigne sur la fonction identitaire de ces lieux. L'acte de venir en famille au musée mobilise des phénomènes de tensions, conciliation, ou de frustration. Ceux-ci découlent des positionnements identitaires - statutaire, intime, familial - des individus par rapport et avec le groupe. Exposant des objets patrimoniaux, le musée a intrinsèquement une mission identitaire mais il participe aussi à la définition de l'identité personnelle et familiale des individus en fournissant un cadre où s'affirme, s'actualise, et se construit le profil culturel de chacun.

\section{... Autour de la construction des rapports des individus à la culture}

Eidelman (2002) a étudié des " carrières de visiteurs », en montrant comment celles-ci s'élaborent de manière continue au cours des différents cycles de la vie, quelle que soit l'initiation muséale reçue pendant l'enfance et la position sociale.

Le contexte familial fait partie de ces « moments » privilégiés de rencontre potentielle des individus avec l'institution. Il offre aux musées un public et en demande. Les familles investissent l'équipement muséal avec de fortes motivations éducatives, visant l'apprentissage cognitif, le développement de la curiosité, de l'esprit critique ou de la pratique. Les objectifs recherchés - réussite scolaire, intégration sociale, épanouissement personnel - révèlent combien ces usages s'inscrivent dans les fonctions de la famille contemporaine. Ceux-ci positionnent le musée comme un lieu d'éducation informelle.

Au-delà de la mobilisation éducative des parents visà-vis de leurs enfants, la visite a aussi une fonction d'éducation auprès des adultes : en famille, certains redécouvrent ou accèdent pour la première fois au musée. Ainsi, les enjeux de ces visites relèvent autant de stratégies éducatives familiales que d'éducation auprès des adultes. La parentalité engendre en tout état de cause une réactualisation du regard sur l'institution.

De cette situation peut naître le désir : chez l'enfant d'une part, mais aussi chez l'adulte, qui, confronté de manière différente au dispositif de l'exposition muséale, peut se trouver étonné, surpris et soudain désireux dans cette relation à l'objet, dans ce rapport au savoir.

\section{... Autour des choix de politique culturelle des musées}

La notion de «public familial » apparaît dans les évaluations d'audience, au début des années 1980, sans être préalablement définie, mais considérée comme une part importante de la fréquentation des musées - impliquant des enjeux culturels et économiques.

Les données qualitatives montrent combien les spécificités de l'institution muséale la préservent pour l'instant de la désaffection des familles et d'une concurrence menaçante des autres types de loisirs. Néanmoins, d'autres activités menées hors du domicile, physiques et sportives notamment, se trouvent en compétition avec la sortie au musée. Durant la journée de visite, ce sont aussi des loisirs physiques, des promenades et d'autres sorties culturelles qui s'y trouvent associées. Au lieu d'opposer la visite aux autres formes de loisirs, il est plus judicieux de les articuler : l'exemple de la Galerie de Paléontologie montre combien les activités de proximité - possibles dans ce cadre particulier (promenade et manège dans le jardin des Plantes, visite de la ménagerie) sont prisées par les familles.

Afin de favoriser les visites familiales et d'en optimiser la qualité, les musées ont tout intérêt à proposer, au-delà des services de restauration et de commodités, la possibilité d'une détente physique, d'une pause conviviale, mais aussi à intégrer à leur offre une autre activité culturelle, interne ou à proximité. On peut imaginer des espaces ludiques intégrant les besoins de décontraction physique des enfants, des espaces extérieurs, et d'autres loisirs culturels particulièrement recherchés par les groupes venant de province souhaitant optimiser leur journée. Les contraintes spatiales n'autorisent pas toujours ce type d'aménagement, des réflexions en termes de réseaux et de partenariats avec d'autres institutions sont à inventer.

Par ailleurs, une autre réflexion se profile quant à l'offre spécifique faite aux familles - offre qui est en augmentation - en termes d'activités (ateliers, visites commentées...) et d'expositions proposées. Dans les deux cas, il s'agit soit de propositions destinées plus particulièrement aux enfants auxquelles l'adulte peut assister, soit de produits conçus à la fois pour les adultes et les enfants avec des contenus croisés ou redondants intégrant les besoins et compétences de chacun. Ainsi, à l'échelle de la visite libre, certains musées présentent des espaces dédiés aux enfants où le parent est positionné comme accompagnateur (c'est le cas par exemple de la Cité des Enfants à la Cité des Sciences et de l'Industrie ou 
encore de la Galerie des Enfants au musée national d'Art moderne...) voire où le parent n'est pas invité, tandis que d'autres musées proposent des expositions à destination des « familles », comme a choisi de le faire le Muséum national d’Histoire naturelle.

Leétude menée auprès des familles n'aboutit pas à recommander une forme d'exposition plus qu'une autre. En réalité, chacune répond à des besoins et motivations familiales différentes : l'enfant a besoin de vivre des expériences personnelles, hors du regard parental, pour se construire lui-même et expérimenter son identité, processus inhérent à la société individualiste ; de même que l'adulte aspire aussi à réaliser des visites à titre personnel et non parental. D'autres dispositifs destinés à l'ensemble du groupe familial répondent quant à eux à l'attente très forte de convivialité et d'éducation, identifiée dans l'étude. Il s'agit en fait de besoins différenciés qui se manifestent et surgissent à différents moments suivant le développement et l'évolution du groupe familial, de ses individus. Il s'agit donc pour chaque musée d'un choix de politique culturelle, choix à faire en connaissance de cause et aussi en fonction - outre des contraintes propres à chaque établissement - des attentes et du profil de ses visiteurs.

\section{Notes}

(1) Cet article est issu d'une recherche en Muséologie sur le public familial des musées : Jonchéry, A. Quand la famille vient au musée : des pratiques de visites aux logiques culturelles. Thèse de doctorat en Muséologie sous la direction de Michel Van Praët, Muséum national d'Histoire naturelle, 2005. La structure même de l'article résume les questionnements et la problématique à l'œuvre dans la recherche doctorale, avant d'en discuter les principaux résultats.

(2) Voir La Déclaration universelle des Droits de l'Homme de 1948 : « la famille est l'élément naturel et fondamental de la société » (article 16).

(3) CPIS : Cadres et Professions Intellectuelles Supérieures

(4) PI : Professions Intermédiaires

\section{Bibliographie}

Benhamou, F. L'économie de la culture. Paris : La Découverte, 2000.

Bourdieu, P., Darbel, A. avec Schnapper, D. L'amour de l'art. Les musées d'art européen et leur public. Paris : Éditions de Minuit, 1966.

Certeau, M. de La culture au pluriel. Paris : Christian Bourgeois Éditeur, $2^{e}$ édition, 1980

Donnat, O. Les pratiques culturelles des Français : enquête 1997. Paris : La Documentation Française, 1998.

Eidelman, J. Musées et publics : la double métamorphose. Thèse d'habilitation à diriger des recherches, Sociologie, CERLIS/CNRS, université Paris V, 2005.

Eidelman, J., Cordier, J-P., Letrait, M. et al. L'espace muséal et ses publics : catégories administratives, catégories de la recherche et catégories "spontanées » des visiteurs. Rapport de recherche en réponse à l'Appel d'offre du département Évaluation et Prospective, CERLIS/ CNRS, 2002.

Eidelman, J., Van Praët, M. (dir.) La muséologie des sciences et ses publics. Paris : PUF, 2000.

Jonchéry, A. La recherche sur les visites et les visiteurs en famille au musée, état de la question, in Familles, écoliers et personnes âgées au musée : Recherches et perspectives. Montréal : ICOM CECA, 2006, pp. 278-318.

Lahire, B. La culture des individus : dissonances culturelles et distinction de soi. Paris : Éditions La Découverte, 2004.

Mironer, L. Cent musées à la rencontre du public. Cabestany : France Édition, 2001.

Niquette, M. Quand les visiteurs ne sont pas seuls : l'analyse sémiocognitive, in La muséologie des sciences et ses publics, J. Eidelman et M. VanPraët (dir.). Paris : PUF, 2000, pp. 181-198.

Passeron, J.-C. Consommation et réception de la culture : la démocratisation des publics, in Le(s) public(s) de la culture, O. Donnat et P. Tolila (dir.). Paris : Presses de Science Po, 2003, pp. 361-390.

Singly, F. de Sociologie de la famille contemporaine. Paris : Nathan, 1999.

Singly, F. de La famille individualiste face aux pratiques culturelles, in Le(s) public(s) de la culture, O. Donnat et P. Tolila (dir.). Paris : Presses de Science Po, 2003, pp. 43-59. 